\title{
Patient Perceptions and Preferences for a Mealtime Insulin Delivery Patch
}

\author{
Mark Peyrot · Darlene Dreon · Vivien Zraick · Brett Cross • \\ Meng H. Tan
}

Received: November 17, 2017 / Published online: January 11, 2018

(C) The Author(s) 2018. This article is an open access publication

\begin{abstract}
Introduction: A basal-bolus insulin regimen is needed to achieve glycated hemoglobin A1c (HbA1c) below $7.0 \%$ in people with type 1 (T1D) or type 2 (T2D) diabetes who have significant loss of beta-cell function. Nonadherence to therapy is common and negatively affects the ability to reach treatment goals. We examined patient assessment of a new, wearable mealtime insulin-delivery system (patch) relative to their current mealtime insulin-delivery system (syringe, pen, or pump). The patch is designed to deliver only boluses of fast-acting insulin (no basal insulin), mechanically controlled by the patient.
\end{abstract}

Methods: Adults $(n=101)$ with T1D or T2D assessed their current mealtime insulin-delivery system and then assessed simulated (no active

Enhanced content To view enhanced content for this article go to http://www.medengine.com/Redeem/ 5E1DF06017C067E3.

\section{Peyrot ( $\square)$}

Department of Sociology, Loyola University

Maryland, Baltimore, MD, USA

e-mail: mark.peyrot@gmail.com

D. Dreon - V. Zraick · B. Cross

Calibra Medical, Wayne, PA, USA

M. H. Tan

Division of Metabolism, Endocrinology and Diabetes, Department of Internal Medicine,

University of Michigan, Ann Arbor, MI, USA medication) patch use over a 3-day period. Participants evaluated mealtime insulin-delivery systems using eight measures from five domains (convenience, interference with daily activities, diabetes-related worry, psychological well-being, and overall satisfaction/preference) on the self-administered Insulin Delivery System Rating Questionnaire. User ratings of their current insulin-delivery systems (syringe, pen, pump) were compared with those for the patch by repeated measure analysis of variance and one-sample $t$ tests.

Results: Participants had significant $(p<0.05)$ preference for patch over syringe in all eight comparisons, and over pen in five out of eight comparisons, with no significant preference for pen. Although there was a preference for patch over pump in six out of eight comparisons, only one showed a significant preference for patch, and one for pump. Significantly more participants reported that they would like to switch to the patch than continue using a syringe (78\% vs $22 \%)$ or pen ( $76 \%$ vs $24 \%$ ) but this difference was not significant for the group using a pump (52\% vs $48 \%$ ).

Conclusions: Participants preferred using the patch over pens and syringes. Its ease of use and discreet method of insulin delivery may contribute to improved patient adherence to mealtime insulin regimens among people currently using injection devices.

Funding: Calibra Medical 
Keywords: Diabetes mellitus; Insulin delivery; Patient preference; Quality of life

\section{INTRODUCTION}

Current clinical practice guidelines by the American Diabetes Association, the European Association for the Study of Diabetes, and Canadian Diabetes Association recommend a goal of glycated hemoglobin A1c (HbA1c) of less than $7.0 \%$ for most people with diabetes [1-3]. This goal is based on the findings of the Diabetes Control and Complications Trial in type 1 diabetes (T1D) [4] and the UK Prospective Diabetes Study in type 2 diabetes (T2D) [5], which report that better glycemic control leads to a lower risk of onset or progression of both microand macrovascular complications. The curvilinear relationship between the microvascular complication rate and HbA1c was almost "flat" in individuals with HbA1c close to 7.0\% [5].

In people without diabetes, glucose homeostasis is attained and maintained by several physiological mechanisms, including continuous basal insulin secretion complemented by prandial insulin peaks when glucose rises after a meal. To achieve glycemic goal, people with T1D need a basal-mealtime insulin regimen, which can be implemented via multiple daily injections (MDI) or continuous subcutaneous insulin infusion (CSII). A basal-mealtime insulin regimen is also often needed to achieve glycemic goal in people with T2D when they develop significant insulin deficiency with progressive loss of beta-cell function.

An estimated $30 \%$ of people with T2D use insulin, either as monotherapy or in combination with oral antihyperglycemic medications to manage their glycemic control [6]. Studies show that insulin therapy is initiated in less than $50 \%$ of people with T2D for whom it is recommended. If insulin treatment is initiated, one-third of people with T2D do not continue with it long term $[7,8]$, and even when treatment is continued, more than half of people with diabetes miss injections, with $20 \%$ admitting to regular omissions of insulin doses [9]. Basal insulin, often taken as a once-a-day injection in the privacy of home during the evening, is less of the barrier to insulin therapy than MDI mealtime insulin [10]. Barriers to MDI adherence include, but are not limited to, the need for multiple injections daily, difficulties associated with insulin administration, lack of discreetness, fear of injection-associated pain, and availability of insulin when needed [11-13].

In the past decade, approximately $60 \%$ of people with diabetes taking insulin did not achieve the proposed glycemic goals [14]. In people with $\mathrm{T} 2 \mathrm{D}$, the prandial insulin response is delayed and blunted, which contributes to postprandial hyperglycemia [15-18]. The closer people with well-controlled T2D are to achieving the HbA1c goal of $7.0 \%$, the more important it becomes to control prandial glucose excursions [19].

However, initiating mealtime insulin and the anticipation of frequent insulin dosing can feel overwhelming to patients. Therefore, there is a need for discreet and easy-to-use insulin delivery systems. Advances have been made in the development of a variety of tools to help patients overcome barriers and simplify insulin delivery, ranging from smaller-gauge needles to minimize injection pain to pre-filled and pre-mixed insulin pens, insulin jet injectors using pressurized air instead of needles to penetrate the skin, insulin pumps to continuously deliver insulin throughout the day, insulin patch-pumps worn attached to the skin for subcutaneous insulin delivery, and a wearable insulin delivery device for on-demand delivery of prandial insulin [20].

Here we discuss a wearable mealtime insulindelivery system (Calibra Medical, Inc., Wayne, PA, USA; in this report referred to as "the patch") that provides a simple, reliable, and discreet option for mealtime insulin delivery. It can be worn on the body for up to 3 days and offers ondemand mealtime insulin for people with diabetes. Insulin is delivered via a subcutaneous cannula that is inserted as the device is applied, which means that the skin is penetrated only once every 3 days rather than multiple times per day, as with mealtime insulin administration via traditional syringe or pen MDI delivery methods.

Similar wearable devices, such as the Omni$\operatorname{pod}^{\circledR}$ Insulin Management System (Insulet Corp, Bedford, MA, USA), V-Go ${ }^{\mathrm{TM}}$ (Valeritas, Inc., Bridgewater, NJ, USA), or PAQ (CeQur ${ }^{\mathrm{TM}}$, 
Montreux, Switzerland), were designed to deliver both basal and bolus insulin doses. Unlike the Calibra patch for bolus insulin delivery (which is completely mechanical and does not require batteries) these other patch-pumps are managed by external controllers or predetermined settings for basal insulin delivery [21].

The aim of this study was to assess user perception, satisfaction, and preferences for their current method of mealtime insulin delivery (syringe, pen, or pump) compared with simulated use (no active medication) of the patch during a 3-day period, using a validated self-report questionnaire.

\section{METHODS}

\section{Study Design}

The study was conducted from 2007 through 2010 at three research centers (Redwood City, CA, USA; Seattle, WA, USA; Orlando, FL, USA). Each participant evaluated the patch after a simulated-use trial period of 3 days at home. They used the patch with sterile diluent only, and in parallel with their usual device for actual mealtime insulin delivery. The Insulin Delivery System Rating Questionnaire (IDSRQ) [22] was administered prior to and after simulated patch use, with the initial administration used to rate the participants' current mealtime insulin-delivery system and the latter used to rate the patch.

The study was approved by the BioMedical Research Institute of America Institutional Review Board (San Diego, CA, USA). All procedures followed were in accordance with the ethical standards of the appropriate committees on human experimentation (institutional and national) and with the Helsinki Declaration of 1964. Informed consent was obtained from all patients for inclusion in the study. All participants provided written informed consent prior to study participation.

\section{Participants}

Participants eligible for the study were 18-85 years of age and had a pre-existing diagnosis of either T1D or T2D. Furthermore, they were required to already use a basal-bolus insulin regimen with a minimum of one basal insulin injection and two mealtime insulin injections per day using syringe or pen, or were on a CSII regimen (insulin pump). Participants were excluded if they were currently employed by a research firm or were working in the medical industry.

\section{Study Device}

The mealtime insulin-delivery system is a small patch $(65 \times 35 \times 8 \mathrm{~mm})$ that can be worn on the body for up to 3 days (Fig. 1). It is classified as a new US Food and Drug Administration product code OPP, under 21 Code of Federal Regulations (CFR) 880.5725; approved for use with rapid-acting insulins Humalog ${ }^{\circledR}$ (Eli Lilly and Co., Indianapolis, IN, USA) and NovoLog ${ }^{\circledR}$ (Novo Nordisk Inc., Plainsboro, NJ, USA). The patch holds up to 200 units of rapid-acting insulin (at 100 units $/ \mathrm{mL}$ ) that are manually administered in increments of 2 units by pressing buttons located on each side of the device. The buttons can be operated through clothing. Mealtime insulin is delivered subcutaneously. After the two buttons are simultaneously pressed, a mechanically timed sequence delivers insulin from the reservoir through a soft polymer cannula into the body (Fig. 2). A built-in alarm (locked buttons) will alert the user if no insulin has been delivered.

\section{Participant Training for Use of the Study Device}

Prior to the simulated-use period, participants received training by a human-factors engineer ("the trainer"), who followed a training protocol developed with a diabetes educator. Patients were trained following the step-by-step instructions outlined in the standard mealtime insulin-delivery system quick-start guide that is provided with the product. After this demonstration, participants completed a comprehension test of the instructions for the tasks of opening the packaging, filling the device, priming the device, applying the device, dosing, 


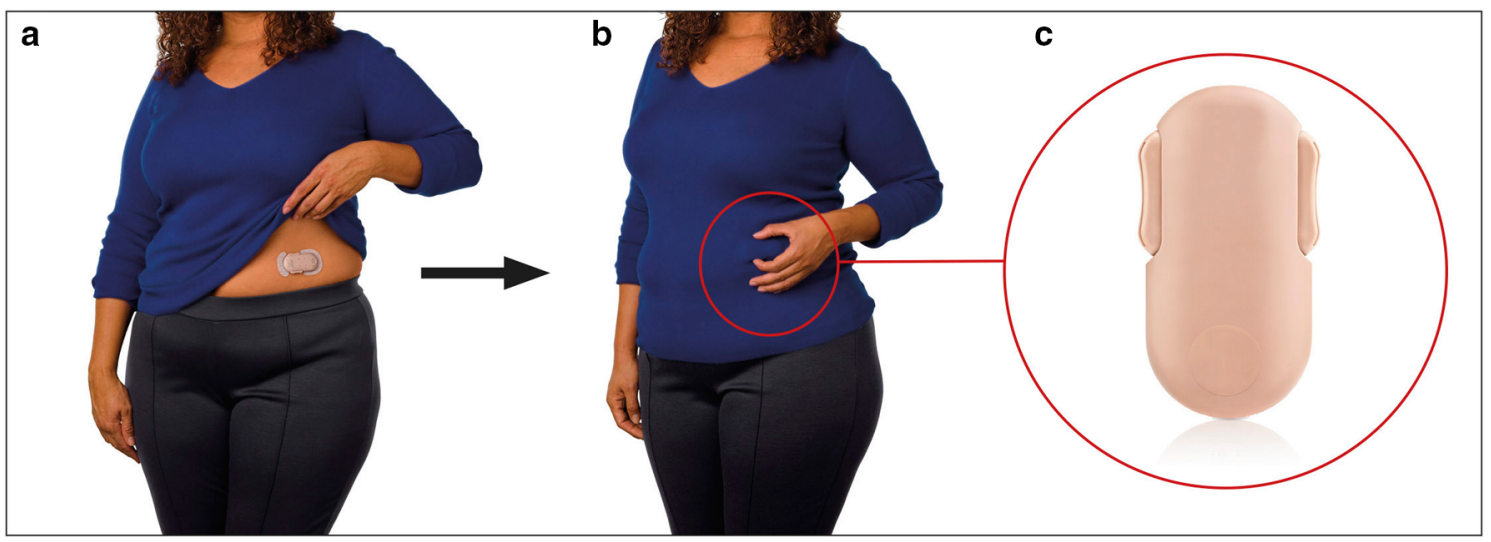

Fig. 1 The wearable on-demand mealtime insulin-delivery system (patch). a The mealtime insulin-delivery patch (Calibra Medical, Inc., Wayne, PA, USA) can be worn for up to 3 days. Mealtime insulin can be dosed discreetly b through clothing by actuating the buttons on both sides of the patch, $\mathbf{c}$ which measures no more than $65 \times 35 \times 8 \mathrm{~mm}$
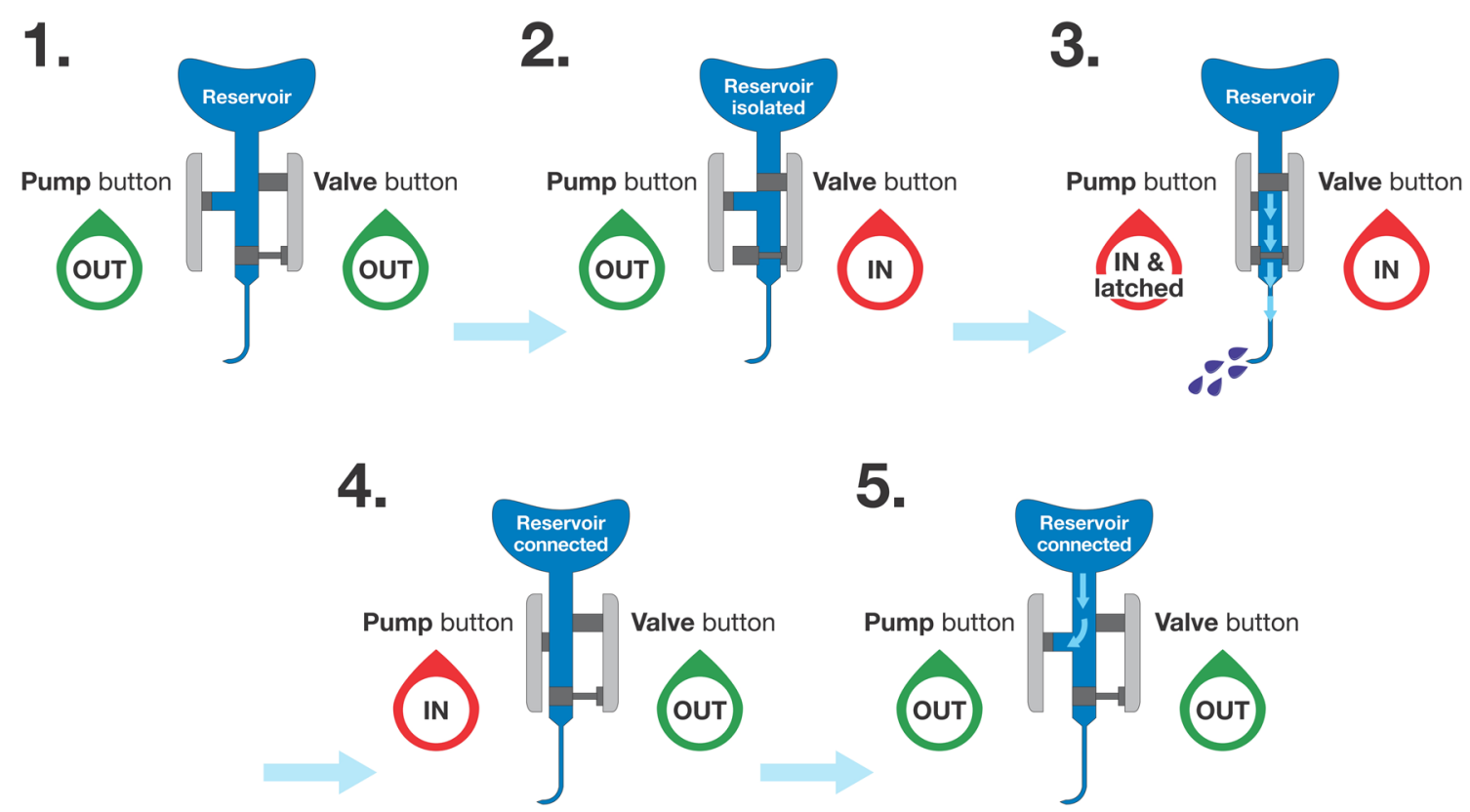

Fig. 2 Schematic of the patch. The mechanically timed sequence ensures that the reservoir feature is never directly connected to the user. Sequence: (1) Both the pump and valve button are "out". (2) Pressing the valve button disconnects the insulin reservoir and opens the pathway to the cannula. (3) Consequently, the pump button depresses, delivering a 2-unit dose. (4) When releasing the buttons,

and removing the device in an open-book test format. Participants then conducted a practice setup and use demonstration under supervision the valve button first returns into the "out" position, closing the pathway to the cannula and reconnecting the insulin reservoir. (5) Finally, the pump button releases and simultaneously draws the next dose from the insulin reservoir. This sequence is repeated for each subsequent dosing

of the trainer and were asked a series of questions to ensure their understanding and safe use of the patch at home. In addition, all 
participants received the standard mealtime insulin-delivery system user guide (provided with the product) containing complete instructions, including all warnings, frequently asked questions, and troubleshooting tips. Participants were advised to carefully read the user guide before beginning to use the patch.

\section{Analytical Measures}

The IDSRQ [22] was used to assess participant self-reported satisfaction with the insulin-delivery system before and after wearing the patch. Participants rated the systems on eight measures in five domains: convenience, interference of treatment with daily activities, diabetes-related worries, psychological well-being (positive and negative), and treatment system satisfaction/ preference (overall satisfaction, compare, switch). The treatment-efficacy subscale was not assessed as respondents did not use the study device to administer insulin to control blood glucose. For the same reason, items related to worry about the efficacy of the insulin-delivery systems were excluded from the measure of diabetes-related worries. Finally, the item regarding cost of the insulin-delivery system was excluded from the convenience measure as there was no cost for simulated use of the study device.

The IDSRQ response options were ordinal scales from 1 to 4 or 1 to 5 and summary measures were calculated as the mean of the items completed for four multi-item subscales (convenience, interference of treatment with daily activities, positive well-being, negative well-being). All IDSRQ measures were converted to scores of 0-100 using standard arithmetic procedure and the small number of missing values was handled as described by Peyrot and Rubin to minimize loss of cases [22].

Two IDSRQ items asked respondents to rate the patch relative to their current treatment system, and therefore were asked only once, after the participant had completed the simulated use of the patch. The first item (compare) was an overall comparison with five response options: 100, "new much better"; 75, "new a bit better"; 50 , "both about the same"; 25 , "current a bit better"; 0 , "current much better." The second item (switch) asked if the participant would like to switch to the patch with four response options: 100 , "definitely yes"; 66.67, "probably yes"; 33.33, "probably not"; 0 , "definitely not."

\section{Statistical Analysis}

Results are reported only for participants who completed evaluations for both their existing and the new device. Descriptive data are reported as percentage for categorical measures or mean [standard deviation (SD)] and range (min-max scores) for continuous measures.

Scores on the six IDSRQ measures of convenience, interference of treatment with daily activities, diabetes-related worry, positive wellbeing, negative well-being, and overall treatment-system satisfaction were analyzed by repeated measures ANOVA comparing ratings of the patch with the ratings of respondents' current mealtime treatment system for those who used either (1) syringe, (2) pen, or (3) pump. Pooled ratings of the patch are reported for all participants; scores for those using each of the current devices are reported as mean [standard error (SE)] of the deviation from the score for the patch (derived separately for respondents who used each particular current device); scores less than zero indicate that the current mealtime insulin-delivery device received a lower score. Effect size was used to show the quantitative strength of each comparison and was calculated as the difference between the means of patch and current device divided by the SD for the rating of the patch by all participants (Cohen's $d$; $d=\frac{(\text { mean score(patch) }- \text { mean score(current device) })}{\mathrm{SD} \text { (patch) }} \quad[23]$; a positive value for $d$ indicates preference for the patch over the current device. Cohen classified descriptors for the magnitude of $d$, where the effect size of $d=0.2$ is considered small, $d=0.5$ is considered a medium effect, and $d=0.8$ is considered a large effect [23]. Thus, if two means differ by no more than $0.2 \mathrm{SD}$, in the absence of objective criteria for assessing the difference it can be concluded that the difference is trivial even if it is statistically significant. If the difference is greater than $0.2 \mathrm{SD}$, it can be considered a nontrivial change even if it is not statistically 
significant (i.e., because of a lack of statistical power). An effect size of $d=0.5$ has been proposed to represent the minimum detectable difference (MDD), a change that is considered recognizable to a careful observer; this concept parallels that of a clinically meaningful difference (CMD) and can be used when there are no objective criteria for assessing CMD [24].

One-sample $t$ tests were used to analyze the two IDSRQ items that asked respondents to rate the patch relative to their current treatment system; deviation of the mean from the midpoint of the item scores was assessed for each of the different current devices (a value of 50 representing equivalence of the patch with the current device).

Two sets of ancillary analyses were performed to identify potential correlates to overall satisfaction with the patch (the latter measure was chosen since it is the summary outcome measure that does not require splitting the sample into subsets, which reduces the number of cases to the point at which correlational analysis is inappropriate). The analyses examined associations between overall satisfaction and (1) all patient characteristics and (2) all domain-specific IDSRQ measures. Preliminary analyses estimated bivariate correlations; if multiple significant correlates were observed, multiple regression analysis was conducted to determine which factors had significant independent associations with overall satisfaction with the patch.

Statistical significance for all analyses was defined as $p \leq 0.05$, two-tailed.

\section{RESULTS}

\section{Study Population}

A total of 101 participants completed the IDSRQ prior to and after simulated use of the patch. Baseline characteristics are summarized in Table 1 . The study population primarily comprised patients with T1D (71\%) and individuals of middle age (mean age 46 years). The mean duration of insulin use was 20 years. Most patients used a syringe $(n=49)$, with the remainder divided between pens $(n=22)$ and CSII $(n=28)$.

\section{Perceptions of Devices}

The four multi-item composite IDSRQ measures all exhibited adequate reliability $(>0.70)$ for the ratings of both the current treatment system and the patch: convenience (Cronbach's $\alpha=0.90 /$ $0.94)$, interference of treatment with daily activities $(\alpha=0.74 / 0.77)$, positive well-being $(\alpha=0.77 /$ $0.81)$, and negative well-being ( $\alpha=0.78 / 0.77)$.

Table 2 reports the results for comparisons of the patch with participants' current mealtime insulin-delivery system. Participants significantly favored the patch over syringe on all eight comparisons $(p<0.05)$, with effect size ranging from 1.49 to 0.41 (median $d=0.70$ ). Participants significantly favored patch over pen for five of eight comparisons (convenience, positive and negative well-being, overall comparison and preference), with no comparisons favoring the pen (effect size ranging from 0.72 to 0.27 , median $d=0.47$ ). Although the patch was favored over the pump on six of eight comparisons, only one significantly favored the patch (negative wellbeing) and one significantly favored the pump (overall comparison); effect size ranged from 0.65 to -0.51 (median $d=0.24$ ).

Significantly more participants reported that the patch was better overall vs their current system for syringe ( $n=37$ vs $n=8)$ and pen ( $n=13$ vs $n=4$ ), but the opposite was true for the pump ( $n=6$ vs $n=18$ ). Significantly more participants reported that they would like to switch to the patch than continue using syringe ( $n=37$ vs $n=10$ ) or pen ( $n=16$ vs $n=5)$, but the difference was not significant for the pump $(n=14$ vs $n=13)$.

\section{Ancillary Analyses}

The analysis examining associations between overall satisfaction and all patient characteristics showed that none were significantly related to overall satisfaction with the patch. The second ancillary analysis showed that four of the five domain-specific IDSRQ measures (all except worry) were related $(p<0.01)$ with the expected sign to overall satisfaction with the patch; the absolute value of the five correlations ranged from 0.17 to 0.59 , with a median of 0.27 . When 
Table 1 Baseline characteristics

\begin{tabular}{ll}
\hline Characteristics & $\begin{array}{l}\text { Participants } \\
(\boldsymbol{N}=\mathbf{1 0 1})\end{array}$ \\
\hline Gender, $n$ (\%) & $51(50)$ \\
Female & $50(50)$ \\
Male & $46 \pm 17(18-85)$ \\
Age, years & \\
Type of diabetes, $n(\%)^{\mathrm{a}}$ & $72(71)$ \\
T1D & $29(28)$ \\
T2D & $25 \pm 17(1-73)$ \\
Age at diagnosis, years & $21 \pm 15(1-67)$ \\
Duration of diabetes, years & $22(22)$ \\
Glucose monitoring (frequency per day), $n(\%)^{\mathrm{b}}$ \\
$<4$ times & $22(22)$ \\
$4-6$ times & $30(30)$ \\
Insulin use, years & $30(30)$ \\
Current device, $n(\%)^{\mathrm{b}}$ & $20 \pm 15(1-67)$ \\
Syringe & \\
Insulin pen & \\
years & \\
\hline
\end{tabular}

Values are shown as mean \pm SD (range), unless indicated otherwise

$S D$ standard deviation, $T 1 D$ type 1 diabetes, $T 2 D$ type 2 diabetes

a May not sum to $100 \%$ because of rounding

b May not sum to $100 \%$ because of missing values

all variables were entered into a stepwise regression analysis, only one (convenience) had an independent association ( $r$-squared $=0.34$, $p<0.001)$.

\section{DISCUSSION}

The patch was well received by the study participants, with only one of 24 comparisons showing a significant advantage for their current insulin treatment system, specifically the pump, possibly because of the participants' established active approaches to their diabetes self-care [25]. After 3 days of simulated patch use, $58 \%$ of syringe or pen users reported that, in terms of overall satisfaction, the patch was better than their current injection system and $12 \%$ indicated it was equal to their current injection system. By contrast, $64 \%$ of pump users reported that the pump was better overall and $14 \%$ indicated it was equal to their current system. Most participants (71\%) reported that they would like to switch to the patch, primarily in preference to syringe and pen.

Patch use resulted in significantly more favorable participant responses for most summary scales when compared with the user's current injection device and was similar to the current insulin-delivery system for all but one of the other comparisons. This reflects findings of a real-life study into the use of this device in people with T1D and T2D [11]. Participants currently using an insulin pen or injecting insulin via a syringe had a more favorable assessment of the patch, indicating a possible preference for an alternative system that does not require multiple daily needle insertions for mealtime insulin.

These favorable responses to the patch may have substantial implications for patient treatment adherence. For people with diabetes, barriers to insulin therapy adherence include the perception that insulin therapy is complicated, fear of hypoglycemia, weight gain, and multiple injections, and the perception that insulin therapy significantly restricts a normal lifestyle $[9,26]$. However, the use of a device that would facilitate discreet insulin delivery and reduce the number of needle-skin penetrations may offer a solution to some of these barriers [27-31]. Compared with traditional MDI insulin regimens, an individual (eating three meals a day) using the patch can potentially decrease the number of mealtime device needle-skin penetrations over 3 days from nine (or more) to one.

This study did not assess objective glucose control or perceived clinical efficacy; however, greater treatment satisfaction (as reported in 
Table 2 Summary of results for device comparison by standardized IDSRQ scores

\begin{tabular}{lllcr}
\hline Measure (number of items) & Patch $^{\mathbf{a}}$ & Syringe $^{\mathbf{b}}$ & Pen $^{\mathbf{b}}$ & \multicolumn{1}{c}{ Pump $^{\mathbf{b}}$} \\
\hline Convenience $^{\mathrm{c}}(11)$ & $78.0 \pm 16.9$ & $-25.3 \pm 4.0^{* * *}(1.49)$ & $-11.9 \pm 3.3^{* *}(0.70)$ & $-4.1 \pm 3.6(0.24)$ \\
Interference $^{\mathrm{d}}(7)$ & $13.4 \pm 15.2$ & $13.4 \pm 3.72^{* * *}(0.88)$ & $7.3 \pm 5.3(0.48)$ & $9.9 \pm 5.0(0.65)$ \\
Worry $^{\mathrm{d}}(1)$ & $37.6 \pm 27.3$ & $18.4 \pm 4.1^{* * *}(0.67)$ & $10.7 \pm 6.8(0.39)$ & $8.3 \pm 5.0(0.30)$ \\
Positive well-being $^{\mathrm{c}}$ (5) & $67.2 \pm 20.3$ & $-11.0 \pm 2.5^{* * *}(0.54)$ & $-8.3 \pm 2.0^{* * *}(0.41)$ & $-4.8 \pm 3.0(0.24)$ \\
Negative well-being $^{\mathrm{d}}(4)$ & $36.7 \pm 19.5$ & $8.0 \pm 2.3^{* * *}(0.41)$ & $9.2 \pm 4.3^{*}(0.47)$ & $9.8 \pm 3.7^{*}(0.50)$ \\
Overall satisfaction $^{\mathrm{c}}(1)$ & $69.4 \pm 23.4$ & $-21.8 \pm 6.6^{* * *}(0.93)$ & $-6.3 \pm 9.1(0.27)$ & $11.9 \pm 6.2(-0.51)$ \\
Device comparison $^{\mathrm{c}, \mathrm{e}}(1)$ & $\mathrm{NA}$ & $-25.0 \pm 4.3^{* * *}(0.73)$ & $-20.2 \pm 6.4^{* *}(0.59)$ & $14.3 \pm 6.1^{*}(-0.42)$ \\
Switching to patch $^{\mathrm{c}, \mathrm{e}}(1)$ & $\mathrm{NA}$ & $-19.5 \pm 4.9^{* * *}(0.61)$ & $-23.0 \pm 5.9^{* * *}(0.72)$ & $-5.6 \pm 5.9(0.18)$ \\
\hline
\end{tabular}

All measures were scored on a scale of $0-100$ to standardize, as described in the original article [22]

$N A$ no independent value for the patch because ratings compare devices, IDSRQ Insulin Delivery System Rating Questionnaire, $S D$ standard deviation, $S E$ standard error

${ }^{*} p \leq 0.05 ;{ }^{* *} p \leq 0.01 ;{ }^{* * *} p \leq 0.001$

${ }^{a}$ Data for the patch are shown as mean $\pm S D$

b Data for syringe, pen, and pump users are shown as mean difference \pm SE relative to the patch (Cohen's $d$, effect size shown as pooled SD units for the patch)

${ }^{c}$ Higher score is better

d Lower score is better

e Test assumes value of 50, representing equal scores for both devices; difference represents deviation from 50

this study) has been associated with better glycemic outcomes [32]. In addition, a clinical study by Bohannon et al. [11] reported that participants using the study device had equivalent mean daily blood glucose and significantly less glycemic variability when compared with using their pen or syringe. The safety profile of this patch has been shown to be similar to currently available injection devices for mealtime insulin [11]. Current literature indicates that more user-friendly and safe-to-use devices, such as insulin pens or pumps, can lead to improved treatment satisfaction and glycemic control compared with using a syringe for insulin delivery $[28,30]$.

This study had several limitations. The use of the patch was simulated; this might create bias toward less favorable patient-reported outcomes because participants, in addition to using the patch, also had to perform insulin administration with their existing mealtime insulin-delivery system. Furthermore, the short duration (3 days) of simulated use did not allow for conclusions regarding long-term patient-reported outcomes to be made. In addition, only user evaluation data was collected, so it was not possible to clinically determine whether use of the patch would impact medication adherence or glucose control.

The study cohort was modest in size, but for the comparison between the current insulindelivery device and the patch, only one effect size larger than the MDD did not reach significance $(d=0.51, p=\mathrm{ns})$, and some effect sizes less than MDD did reach significance (e.g., $d=0.51, p>0.001)$. This suggests that sample size was not a problem, as meaningful differences in patient-reported outcomes should be recognizable by those making the ratings. Patient characteristics were not significantly correlated with the key rating of the patch, suggesting that observed perceptions of the study device are robust and it was unnecessary for our analysis to control for case-mix variables. However, future research should examine the degree to which patient characteristics, 
including regimen intensity, are related to perceptions of the study device in actual clinical practice.

It also is worth noting that the device is currently labeled to be worn on the abdomen. In addition, although the age distribution of participants in this study was $18-85$ years, the device is currently labeled for use by patients over 21 years of age.

Physicians may be reluctant to initiate or intensify insulin treatment in people with T2D despite recognizing the need for it (a phenomenon known as "clinical inertia"). Physicians are concerned that patients will be unable to follow the insulin-treatment regimen [33]. There is also a concern that training people with diabetes to properly calculate insulin doses and administer insulin correctly is too complicated and time-consuming [34]. Future studies should examine physicians' reactions to the study device to determine whether the use of a system that is discreet to wear and use and requires fewer daily needle-skin penetrations might have an impact on prescribing behaviors and reduce clinical inertia.

\section{CONCLUSIONS}

The patch evaluated here is a small and easy-touse device that allows for discreet delivery of mealtime insulin. While the system does not eliminate the need for daily basal-insulin injections, it is intended to assist in mealtime dosing by providing an alternative to the multiple daily injections required with syringe or pen devices. Rather than having to penetrate the skin multiple times per day, application of the patch requires only one skin penetration every 3 days. Given that a mealtime dose of insulin from this device is not associated with a needle-skin penetration, use of the patch may help increase patient adherence to multipledaily-insulin-injection regimens, not only because of user satisfaction with the patch but also reductions in the patient burden associated with MDI. Use of this patch may lead to better control of prandial hyperglycemic excursions and improved $\mathrm{HbA1c}$ in those people with diabetes who miss mealtime insulin injections with pen or syringe. Research is needed to examine this possibility.

\section{ACKNOWLEDGEMENTS}

Funding. The studies were funded by Calibra Medical. The study sponsor, Calibra Medical (Wayne PA), was involved in all stages of research and manuscript preparation; all authors had access to the data, and contributed to the manuscript development. Calibra Medical funded the article processing charges.

Authorship. All named authors meet the International Committee of Medical Journal Editors (ICMJE) criteria for authorship for this manuscript, take responsibility for the integrity of the work as a whole, and have given final approval for the version to be published. The authors would like to thank Brian L. Levy, MD, and Ramachandra G. Naik, MD for their critical feedback.

Medical Writing Assistance. Editorial and writing support in the preparation of this manuscript was provided by Martina Fuchsberger, PhD, of Excerpta Medica, funded by Calibra Medical.

Disclosures. Mark Peyrot has received consulting fees from Calibra Medical. Meng H. Tan has received consulting fees from Calibra Medical. Darlene Dreon is a full-time employee of Calibra Medical. Vivien Zraick is a former fulltime employee of Calibra Medical. Brett Cross is a former full-time employee of Calibra Medical.

Compliance with Ethics Guidelines. All procedures followed were in accordance with the ethical standards of the appropriate committees on human experimentation (institutional and national) and with the Helsinki Declaration of 1964. Informed consent was obtained from all patients for inclusion in the study.

Data Availability. The datasets generated and/or analyzed during the current study are 
available from the corresponding author on reasonable request.

Open Access. This article is distributed under the terms of the Creative Commons Attribution-NonCommercial 4.0 International License (http://creativecommons.org/licenses/ by-nc/4.0/), which permits any noncommercial use, distribution, and reproduction in any medium, provided you give appropriate credit to the original author(s) and the source, provide a link to the Creative Commons license, and indicate if changes were made.

\section{REFERENCES}

1. American Diabetes Association. Standards of medical care in diabetes. 5. Glycemic targets. Diabetes Care. 2016;39(Suppl 1):S39-46.

2. Inzucchi S, Bergenstal R, Buse J, et al. Management of hyperglycaemia in type 2 diabetes, 2015: a patient-centred approach. Update to a position statement of the American Diabetes Association and the European Association for the Study of Diabetes. Diabetologia. 2015;58:429-42.

3. Imran SA, Rabasa-Lhoret R, Ross S. Canadian Diabetes Association 2013 clinical practice guidelines for the prevention and management of diabetes in Canada: targets for glycemic control. Can J Diabetes. 2013;37(Suppl 1):S31-4.

4. The Diabetes Control and Complications Trial (DCCT) Research Group. The effect of intensive treatment of diabetes on the development and progression of long-term complications in insulindependent diabetes mellitus. $\mathrm{N}$ Engl J Med. 1993;329:977-86.

5. UK Prospective Diabetes Study (UKPDS) Group. Intensive blood-glucose control with sulphonylureas or insulin compared with conventional treatment and risk of complications in patients with type 2 diabetes (UKPDS 33). Lancet. 1998;352:837-53.

6. National Diabetes Statistics Report. 2014. http:// www.cdc.gov/diabetes/pubs/statsreport14/nationaldiabetes-report-web.pdf. Accessed Sept 15, 2016.

7. Roussel R, Charbonnel B, Behar M, Gourmelen J, Emery C, Detournay B. High rates of early discontinuation of insulin therapy in type 2 diabetes: a nationwide study. Diabetes. 2015;64(Suppl 1):A252.
8. Karter AJ, Subramanian U, Saha C, et al. Barriers to insulin initiation: the translating research into action for diabetes insulin starts project. Diabetes Care. 2010;33:733-5.

9. Peyrot M, Rubin RR, Kruger DF, Travis LB. Correlates of insulin injection omission. Diabetes Care. 2010;33:240-5.

10. Peyrot M, Barnett AH, Meneghini LF, SchummDraeger PM. Insulin adherence behaviours and barriers in the multinational Global Attitudes of Patients and Physicians in Insulin Therapy study. Diabet Med. 2012;29:682-9.

11. Bohannon N, Bergenstal $\mathrm{R}$, Cuddihy $\mathrm{R}$, et al. Comparison of a novel insulin bolus-patch with pen/syringe injection to deliver mealtime insulin for efficacy, preference, and quality of life in adults with diabetes: a randomized, crossover, multicenter study. Diabetes Technol Ther. 2011;13:1031-7.

12. Rubin RR. Adherence to pharmacologic therapy in patients with type 2 diabetes mellitus. Am J Med. 2005;118(Suppl 5A):S27-34.

13. Kruger DF, LaRue S, Estepa P. Recognition of and steps to mitigate anxiety and fear of pain in injectable diabetes treatment. Diabetes Metab Syndr Obes. 2015;8:49-56.

14. Giugliano D, Maiorino MI, Bellastella G, Chiodini P, Ceriello A, Esposito K. Efficacy of insulin analogs in achieving the hemoglobin A1c target of $<7 \%$ in type 2 diabetes: meta-analysis of randomized controlled trials. Diabetes Care. 2011;34:510-7.

15. Vinik AI, Kalk WJ, Botha JL, Jackson WP, Blake KC. The inexhaustible beta cell. Diabetes. 1976;25:11-5.

16. Service FJ, Hall LD, Westland RE, et al. Effects of size, time of day and sequence of meal ingestion on carbohydrate tolerance in normal subjects. Diabetologia. 1983;25:316-21.

17. Cerasi E, Luft R. The plasma insulin response to glucose infusion in healthy subjects and in diabetes mellitus. Acta Endocrinol (Copenh). 1967;55:278-304.

18. Del Prato S. Loss of early insulin secretion leads to postprandial hyperglycaemia. Diabetologia. 2003;46(Suppl 1):M2-8.

19. Monnier L, Lapinski H, Colette C. Contributions of fasting and postprandial plasma glucose increments to the overall diurnal hyperglycemia of type 2 diabetic patients: variations with increasing levels of HbA(1c). Diabetes Care. 2003;26:881-5. 
20. Varshney HM, Kumar R, Mohan S. Novel approaches for insulin delivery: current status. Int J Ther Appl. 2012;7:25-31.

21. Anhalt $\mathrm{H}$, Bohannon NJV. Insulin patch pumps: their development and future in closed-loop systems. Diabetes Technol Ther. 2010;12(Suppl 1):S51-8.

22. Peyrot M, Rubin RR. Validity and reliability of an instrument for assessing health-related quality of life and treatment preferences: the Insulin Delivery System Rating Questionnaire. Diabetes Care. 2005;28:53-8.

23. Cohen J. Statistical power analysis for the behavioral sciences. 2nd ed. Hillsdale: Lawrence Earlbaum Associates; 1988.

24. Norman GR, Sloan JA, Wyrwich KW. Interpretation of changes in health-related quality of life: the remarkable universality of half a standard deviation. Med Care. 2003;41:582-92.

25. Ritholz MD, Smaldone A, Lee J, Castillo A, Wolpert $\mathrm{H}$, Weinger K. Perceptions of psychosocial factors and the insulin pump. Diabetes Care. 2007;30:549-54.

26. Summers KH, Szeinbach SL, Lenox SM. Preference for insulin delivery systems among current insulin users and nonusers. Clin Ther. 2004;26:1498-505.

27. Kerr D, Hoogma RP, Buhr A, Petersen B, Storms FE, Study investigators. Multicenter user evaluation of ACCU-CHEK ${ }^{\circledR}$ Combo, an integrated system for continuous subcutaneous insulin infusion. J Diabetes Sci Technol. 2010;4:1400-7.
28. Klausmann G, Hramiak I, Qvist M, Mikkelsen KH, Guo X. Evaluation of preference for a novel durable insulin pen with memory function among patients with diabetes and health care professionals. Patient Prefer Adherence. 2013;7:285-92.

29. Mader JK, Lilly LC, Aberer F, et al. A feasibility study of a 3-day basal-bolus insulin delivery device in individuals with type 2 diabetes. Diabetes Care. 2014;37:1476-9.

30. Barnard KD, Bromba $M$, de Lange $M$, et al. High reported treatment satisfaction in people with type 1 diabetes switching to latest generation insulin pump regardless of previous therapy. J Diabetes Sci Technol. 2015;9:231-6.

31. Berard L, Cameron B, Woo V. Pen needle preference in a population of Canadians with diabetes: results from a recent patient survey. Can J Diabetes. 2015;39:206-9.

32. Hanestad BR, Albreksten G. Quality of life, perceived difficulties in adherence to diabetes regimen, and blood glucose control. Diabet Med. 1991;8:759-64.

33. Polinski JM, Smith BF, Curtis BH, et al. Barriers to insulin progression among patients with type 2 diabetes: a systematic review. Diabetes Educ. 2013;39:53-65.

34. Sorli C, Heile MK. Identifying and meeting the challenges of insulin therapy in type 2 diabetes. J Multidiscip Healthc. 2014;7:267-82. 